\title{
Yu Nu Compound Regulates Autophagy and Apoptosis Through mTOR in vivo and vitro
}

This article was published in the following Dove Press journal:

Diabetes, Metabolic Syndrome and Obesity: Targets and Therapy

Caigu He'
Guang Liu'
Shuting Zhuang
Jialin Zhang
Yangtao Chen'
Hetian Li'
Zhengping Huang
Yanfang Zheng
'Department of Histology and
Embryology, Fujian University of
Traditional Chinese Medicine, Fuzhou,
Fujian 350I22, People's Republic of
China; ${ }^{2}$ Department of Biochemistry,
Fujian University of Traditional Chinese
Medicine, Fuzhou, Fujian 350I22,
People's Republic of China; ${ }^{3}$ Department
of Pharmacology, Fujian University of
Traditional Chinese Medicine, Fuzhou,
Fujian 350I22, People's Republic of China

Correspondence: Caigu He Fujian University of Traditional Chinese Medicine, People's Republic of China Email caiguheeel@I63.com
Introduction: $\mathrm{Yu} \mathrm{Nu}$ compound (YNJ) is a traditional Chinese medicine widely utilized to treat type 2 diabetes possibly through mediating autophagy. Abnormal podocyte autophagy and apoptosis could result in podocyte loss in diabetics nephropathy (DN). The mechanism of $\mathrm{Yu} \mathrm{Nu}$ compound in DN is still unclear. Therefore, the study aims to investigate the effects of $\mathrm{Yu} \mathrm{Nu}$ compound and analyze the potential mechanism.

Methods: Goto-Kakizaki (GK) rats were administered using YNJ with different doses once a day by gavage for 4 weeks. The renal cortex injury was observed by HE staining and electron microscope. Cell apoptosis of renal cortex was analyzed by TUNNEL staining. The mTOR, autophagy-related proteins and apoptosis-related proteins were detected by Western blot or real-time PCR in vivo and vitro. MPC5 cells were exposed to high glucose (HG, $30 \mathrm{mM}$ ) for $12 \mathrm{~h}$ to simulate podocyte injury in DN. MPC5 cells were treated by serum containing YNJ with different dosages. Cell activities and apoptosis were, respectively, detected through Cell Counting Kit-8 (CCK8) assay and flow cytometry.

Results: The results showed that the medium dose of YNJ had better effects on decreasing blood glucose and improving renal injury in GK rats, followed by decreasing mTOR levels. The autophagy levels were enhanced in renal cortex, accompanied with the increase of cell apoptosis in vivo. Besides, the proteins regulating autophagy and apoptosis were significantly modulated by YNJ in GK rats. Then, we found that the decreasing endogenous mTOR could reverse the effects of YNJ on podocyte apoptosis and autophagy in vivo.

Discussion: The study suggested that YNJ recovered normal autophagy and suppressed apoptosis through regulating mTOR. The maintenance of normal basal autophagic activity possibly based on the effect of YNJ on multiple target was essential for maintaining podocyte function.

Keywords: traditional Chinese medicine, diabetics nephropathy, LC3, Bcl-2, mTOR

\section{Introduction}

$\mathrm{Yu} \mathrm{Nu}$ compound is a traditional Chinese medicine that has significant therapeutic effects for patients with type 2 diabetes. ${ }^{1}$ A study demonstrates that YNJ could regulate autophagy and apoptosis to reducing cell injury in diabetes. ${ }^{2}$ Autophagy plays considerable roles in the growth and development of podocytes. ${ }^{3,4}$ Podocytes are highly differentiated cells that belong to outer part of the glomerular basement membrane and form the last defence in the glomerular filtration barrier. Podocytes injury could disrupt the integrity of the filter membrane and cause proteinuria. Autophagy is a process that engulfs its own cytoplasmic proteins or organelles into vesicles. Then, vesicles fuses with lysosomes to form autophagic lysosomes (autophagolysosome) which degrades the contents it contains. Autophagy can remove damaged or aging organelles and biomacromolecule, which is common in 
eukaryocyte, but the level of autophagy in most cells is low. ${ }^{5}$ Autophagy is complex in the pathogenesis of diabetes. ${ }^{6}$ Early exposure of high glucose (HG) could induce podocytes autophagy. ${ }^{7,8}$ However, the autophagy levels were reduced with the prolonged glucose exposure time. ${ }^{8}$ Podocytes death would occur when cell autophagy and apoptosis continue to show upregulation. Therefore, autophagy showed different effects with the time of cell exposure to HG. In contrast, cell apoptosis gradually increases under exposure to $\mathrm{HG}^{9}$ A study has shown that autophagy is the initiator that triggers the apoptosis. ${ }^{10}$ There are studies revealing that HG triggers mitochondriadependent apoptosis pathway in DN and autophagy induces cell apoptosis independent of Bim mediating pathway, which belongs to one of Bcl-2 family protein members. ${ }^{11}$ Once autophagy is activated, LC3-I partakes in ubiquitin-like reaction and forms lipidized form of LC3 (LC3II) as structural proteins of autophagosomes. Atg12 and Atg5 play vital roles in the extension of autophagy. ${ }^{12}$ mTOR exists in mTOR1 and mTOR2 form in cells. The current studies have suggested that the pathogenesis of diabetes is related to autophagy inhibition caused by activation of the mTOR signaling pathway. ${ }^{13}$ mTOR pathway is involved in regulating autophagy and apoptosis pathway in $\mathrm{DN}^{14,15}$ Besides, mTOR pathway is implicated in autophagy and apoptosis of podocytes exposed to HG. ${ }^{16}$ Also, mTOR phosphorylates autophagy-related protein to suppress initiation of autophagy. ${ }^{17}$ Therefore, the study aimed to investigate how $\mathrm{Yu} \mathrm{Nu}$ compound exerted functions in DN.

\section{Methods}

\section{Animals}

GK rats of Specific pathogen Free (SPF) $(n=45$, age: 10 weeks, weight: $320 \pm 22 \mathrm{~g}$ ) were purchased (CAVENS, Changzhou, China. number of animal license: SCXK 2016-0010. Certificate number: 20,170,005,000,503). Wistar rats of SPF were purchased (Shanghai slack laboratory animal co. LTD, Shanghai, China. number of animal license: SCXK 2017-0005. Certificate number: 201,827,392). The rats were raised in Fujian university of traditional Chinese medicine laboratory animal center barrier system. After being fed adaptively for 4 weeks, the diabetic rats were determined through testing the random blood glucose beyond $11.1 \mathrm{mmol} / \mathrm{L}$. The GK rats were divided into $\mathrm{Yu}$ $\mathrm{Nu}$ compound group (YNJ), metformin group and model group (GK). Seven Wistar rats of the same strain and age were used as normal group (control). Metformin group was treated intragastrically by metformin (100mg.kg-1 $\left.\mathrm{d}^{-1}\right)$. The model and normal group were administered by gavage with normal saline. Simultaneously, the YNJ group was given an appropriate dose according to body weight once a day for 4 weeks. Yu Nu compound consisted of gypsum, rehmannia, Radix Ophiopogonis, anemarrhenae and bidentata root. The rats were administered by 6.25 times dose of adults (gypsum: 15g, rehmannia: 30g, Radix Ophiopogonis: 6g, anemarrhenae: $4.5 \mathrm{~g}$, bidentata root: $4.5 \mathrm{~g}$ ). Thus, the dose of $\mathrm{Yu} \mathrm{Nu}$ compound used by the rats was: gypsum $1.56 \mathrm{~g} \cdot \mathrm{kg}^{-1} \cdot \mathrm{d}{ }^{-1}$ rehmannia: $\quad 3.125 \mathrm{~g} \cdot \mathrm{kg}^{-1} \cdot \mathrm{d}^{-1} \quad$ Radix Ophiopogonis: $0.625 \mathrm{~g} \cdot \mathrm{kg}^{-1} \cdot \mathrm{d},^{-1}$ anemarrhenae: $0.469 \mathrm{~g} \cdot \mathrm{kg}^{-1} \cdot \mathrm{d}^{-1}$ and bidentata root $0.469 \mathrm{~g} \cdot \mathrm{kg}^{-1} \cdot \mathrm{d}$. $^{-1}$ The compounds were oaked into $1000 \mathrm{~mL}$ water for $2 \mathrm{~h}$ and boiled together. The fluid was kept after simmer for 15 minutes. The remaining was added into $500 \mathrm{~mL}$ water, followed by decoction to get fluid. The two fluids were mixed and condensed mixture of $1.25 \mathrm{mg} / \mathrm{mL}$, which was conserved at $4^{\circ} \mathrm{C}$. The GK rats in $\mathrm{YNJ}$ group were divided into YNJH (high dose): 12.5 g.kg-1.d-1, YNJM (medium dose): 6.25g. kg-1.d-1, YNJL (low dose):3.125g. $\mathrm{kg}-1 . \mathrm{d}-1$. The GK rats in different groups were separately administered intragastrically using the different dose of YNJ for consecutive 4 weeks. After final administration, the rats were anaesthetized using $1 \%$ pentobarbital $(40 \mathrm{mg} / \mathrm{kg})$ for collecting renal cortex. The part of renal cortex was put into $2.5 \%$ glutaraldehyde and $1.5 \%$ paraformaldehyde for the preparation and observation of electron microscope specimens. Besides, the part of renal cortex was fixed using $4 \%$ paraformaldehyde for HE staining. The rest was stored at $-80^{\circ} \mathrm{C}$ for Real-time PCR and Western blot. The study was approved by Laboratory Animal Ethics Committee of Fujian University of Traditional Chinese Medicine, followed the Guidelines of the Care and Use of Laboratory Animals issued by the Chinese Council on Animal Research.

\section{HE Staining}

The renal cortex sections were deparaffined with xylene and dehydrated using gradient alcohol. The sections were stained using Hematoxylin for $15 \mathrm{~min}$. Then, after hydrochloric acid alcohol differentiation, the sections were washed with water for $10 \mathrm{~min}$ and next stained by Eosin stain for $1 \mathrm{~min}$, which then was sealed using Neutral balsam. The sections were observed and photographed under Nikon 55i microscope.

\section{Electron Microscope}

Renal cortex was fixed using 3\% glutaraldehyde, $1.5 \%$ paraformaldehyde and $0.1 \mathrm{M}$ PBS (PH 7.2) at $4^{\circ} \mathrm{C}$ 
overnight, following washing with $0.1 \mathrm{M}$ PBS for three times, $30 \mathrm{~min}$ each. The renal tissue was fixed. The $1 \%$ osmium and $1.5 \%$ potassium ferricyanide at $4^{\circ} \mathrm{C}$ for $1.5 \mathrm{~h}$. The renal tissue was dehydrated through a series of process including $50 \%$ alcohol for $10 \mathrm{~min}, 70 \%$ alcohol saturated uranium acetate dye at $4{ }^{\circ} \mathrm{C}$ overnight, $90 \%$ alcohol for $10 \mathrm{~min}, 90 \%$ alcohol-acetone for $10 \mathrm{~min}, 90 \%$ acetone for $10 \mathrm{~min}$ and anhydrous acetone for $10 \mathrm{~min}$. Then, the renal tissue was soaked in anhydrous acetone and epoxy resin 618 embedding agent (1:1) for $1.5 \mathrm{~h}$, followed by epoxy resin 618 embedding agent at $35^{\circ} \mathrm{C}$ for $3 \mathrm{~h}$. Finally, the tissue was stained by uranyl acetate and lead citrate, respectively, for $10 \mathrm{~min}$ after being embedded, polymerized and sliced $(70-80 \mathrm{~nm})$.

\section{Real-Time PCR}

The total RNA of renal cortex was extracted using Trizol, the concentration of which was detected using ultraviolet spectrophotometer. The RNA was reversed into cDNA using Reverse transcription kit. The primers were designed using Primer Premier 5.0 software ( $\beta$-actin R:5' TAG AGGTCTTTACGGATGTCAACGT3'; ATG12 F: 5' GCT GAAGGCTGTAGGAGACACT3', M-TOR F: 5' ACGAG TTTGTTTTCCTCCTGAA3', M-TOR R: 5' AGAGAA GTTGGGTCATTGGCTA3'). The mRNA was amplified in ABI StepOne plus PCR instrument and their levels were calculated using $2^{-\Delta \Delta \mathrm{Ct}}$.

\section{Western Blot}

The renal cortex tissue and podocytes were lysed with RIPA Lysis Buffer, which then was centrifuged at $12000 \mathrm{r} / \mathrm{min}$ for $15 \mathrm{~min}$ at $4^{\circ} \mathrm{C}$. The protein concentration was detected using BCA method. The proteins were separated through electrophoresis and transferred into nitrocellulose membrane. The primary antibodies (anti-ATG12, Anti-ATG5, anti-mTOR, anti-Bcl-2, anti-Bax, anti-Caspase-3, anti-GADPH, 1:1000, Abcam, England) (anti-Beclin-1, anti-LC3II: 1:1000. Santa Cruz, America) (Abcam, America) (Cleaved-caspase3: 1:500, Abcam, America) were incubated with the membrane at $4^{\circ} \mathrm{C}$ overnight blocked by $5 \%$ skim milk. The secondary antibody (1:5000) was then incubated with membrane for $2 \mathrm{~h}$. The gray value of protein was analyzed using Image Lab 3.0 software and the relative expression was calculated in the ratio of the target protein and reference.

\section{TUNNEL Staining}

The paraffine sections of renal tissue were waxed with xylene and dehydrated by gradient using ethanol. The protease $\mathrm{K}(20 \mu \mathrm{g} / \mathrm{mL})$ containing no DNase was dropped into the sections at $20^{\circ} \mathrm{C}$ for $30 \mathrm{~min}$. Then, the sections were washed using PBS twice. The sections were incubated with TUNNEL solution $(50 \mu \mathrm{L}$, Abcam, America) at $37^{\circ} \mathrm{C}$ for $1 \mathrm{~h}$ and then observed under a fluorescent microscope.

\section{Cell Line}

MPC5 cells (MPC5, ATCC, USA) were purchased by MINGJING BIOLOGY (Shanghai, China), the agent of ATCC (USA). Cells were cultured in RPMI1640 medium containing $10 \%$ fetal bovine serum at $37^{\circ} \mathrm{C}$ with $5 \% \mathrm{CO}_{2}$. INF- $\gamma$ was used to induce cells proliferation. When cells grew to $80 \%$ confluency, cells were digested with trypsin and used to passage. Then, cells were cultured in medium containing no INF- $\gamma$ at $37^{\circ} \mathrm{C}$ with $5 \% \mathrm{CO}_{2}$, which was differentiated and matured in 10-14 days for further experiment. The cells were treated by high glucose $(30 \mathrm{mmol} / \mathrm{L})$, high glucose + YNJ-Low: $1 \mathrm{mg} / \mathrm{mL}$, high glucose + YNJ-Mid: $2 \mathrm{mg} / \mathrm{mL}$, or high glucose + YNJ-High: $4 \mathrm{mg} / \mathrm{mL}$ for $12 \mathrm{~h}$.

\section{The Preparation of Serum Containing Drug}

The rats were administered by gavage using aforementioned drug once a week. The blood was collected by abdominal aorta under anesthesia $2 \mathrm{~h}$ after final administration. After clotting, the serum was collected at 3000r/ min for $10 \mathrm{~min}$, which then was put in the $56^{\circ} \mathrm{C}$ water for $30 \mathrm{~min}$. The serum was filtered and sterilized with disposable sterile filter of $0.22 \mu \mathrm{m}$, which then was stored in $-20^{\circ} \mathrm{C}$ for further experiments.

\section{CCK8 Assay}

The cells were seeded into 96-well plate $\left(5^{*} 10^{3} / \mathrm{mL}\right)$ and pre-cultured for $24 \mathrm{~h}$ at $37^{\circ} \mathrm{C}$ with $5 \% \mathrm{CO}_{2}$. DN cell models were divided into five groups (control, model, YNJ-Low: $1 \mathrm{mg} / \mathrm{mL}$, YNJ-Mid: $2 \mathrm{mg} / \mathrm{mL}$, YNJ-High: $4 \mathrm{mg} / \mathrm{mL}$ ). Each group was set 5 double wells. $12 \mathrm{~h}$ after intervention, CCK8 solution $(10 \mu \mathrm{L}$, DOJINDO, Shanghai, China) was added to incubate the cells for $2 \mathrm{~h}$. The OD at $450 \mathrm{~nm}$ was detected by microplate reader.

\section{Flow Cytometry}

The MPC5 cells were washed with PBS and digested using $0.25 \%$ trypsin. The supernatant was discarded after centrifugation at $1000 \mathrm{r} / \mathrm{min}$ and PBS was added into cells. The cell suspension $\left(1^{*} 10^{6}\right.$ cells $)$ was collected and 
centrifuged at 1000r/min for $5 \mathrm{~min}$. $500 \mu \mathrm{L}$ binding buffer was added after the supernatant was discarded. $5 \mu \mathrm{L}$ Annexin V-FITC and $5 \mu \mathrm{L}$ propidium iodide were respectively added into cell suspension, which was incubated and kept in dark place for 10min. The cell apoptotic levels were analyzed through flow cytometry (Beckmancoulter, America).

\section{Plasmid Transfection}

Cells in logarithmic growth were digested using trypsin and seeded into 24 well-plate. When cells grew to $70 \%$. The mTOR interference plasmids (shRNA-mTOR) shRNA-mTOR or shRNA-NC were transfected into cells by Lipofectamine ${ }^{\mathrm{TM}} 2000$ according to manufacture's protocols (Invitrogen, America). 4-6 h after transfection, the cells were cultured in the medium containing no $\gamma$-IFN at $37^{\circ} \mathrm{C}$ with $\mathrm{CO}_{2}$. Further experiments in cells were performed after transfection of $24 \mathrm{~h}$. The cells were exposed to $\mathrm{HG}$ or YNJ-Mid for $12 \mathrm{~h}$.

\section{Statistical Analysis}

The data were shown as mean \pm SD. The comparison among different groups was performed by one-way ANOVA, followed by Turkey's test. $\mathrm{P}<0.05$ was considered as statistically significant.

\section{Results}

\section{Yu Nu Compound Significantly Reduced the Blood Glucose (BG) in GK Rats}

The random blood glucose of GK rats presented progressive increase to more than $20 \mathrm{mmol} / \mathrm{L}$ with the age (Table 1). BG in Metformin group was significantly decreased one week after administration comparing to GK group. However, BG showed significant decrement two weeks after YNJM administration comparing to GK group and BG presented similar effects after five weeks of YNJH administration. The inhibitory effects of YNJM on BG were significantly superior to other doses. Thus, the medium dose of YNJ had better effects on decreasing BG.

Table 1 The blood glucose in different time after YJN administration. Data were shown as mean \pm SD. ${ }^{*} p<0.05$, $* * p<0.01$ or $* * * p<0.001$ comparing to Control group. ${ }^{\Delta} p<0.05,{ }^{\Delta \Delta} p<0.01$, or ${ }^{\Delta \Delta \Delta} p<0.001$.

\section{Yu Nu Compound Improved Renal Injury}

Capsule and tubular lumen presented obvious enlargement in GK group than control group. Furthermore, the lining cells of renal capsule transited from monolayer flat cells to monolayer cuboidal cells comparing to control. There were no obvious differences in the morphology of renal corpuscles and tubules between metformin and normal. Comparing to control group, capsular space and renal tubular lumen showed enlargement in YNJH. Vascular spherule atrophied, renal capsule lumen showed enlargement, and the lining cells of renal capsule migrated to monolayer cubic occasionally in YNJL. HE staining indicated that the effects of YNJM on improving renal injury were better than other YNJ group (Figure 1).

\section{Comparison of Renal Ultrastructure in Each Group}

In control group, the endothelial structure of fenestrated capillary in renal corpuscle was clear and the podocyte protuberances enclosed the basement membrane of the capillary endothelium (Figure 2). The basal membrane thickness in the GK group was significantly higher than control group (Table 2). Moreover, the fenestration of endothelial cells was relatively disordered. There were more autophagosomes in the cytoplasm of the podocytes, and more vacuolated structures in the epithelial cells of the

Table I The Comparison of Random Blood Glucose Levels in Each Group (Mean \pm SD)

\begin{tabular}{|c|c|c|c|c|c|c|c|}
\hline \multirow[t]{2}{*}{ Group } & \multirow[t]{2}{*}{$\mathbf{n}$} & \multicolumn{6}{|c|}{ Random Blood Glucose (mmol/L) } \\
\hline & & $\begin{array}{l}\text { Before } \\
\text { Administration }\end{array}$ & $\begin{array}{l}\text { After Iw of } \\
\text { Administration }\end{array}$ & $\begin{array}{l}\text { After } 2 \mathrm{w} \text { of } \\
\text { Administration }\end{array}$ & $\begin{array}{l}\text { After } 3 w \text { of } \\
\text { Administration }\end{array}$ & $\begin{array}{l}\text { After } 4 \mathrm{w} \text { of } \\
\text { Administration }\end{array}$ & $\begin{array}{l}\text { After } 5 \mathrm{w} \text { of } \\
\text { Administration }\end{array}$ \\
\hline Control & 7 & $6.26 \pm 0.40$ & $6.56 \pm 0.74$ & $6.77 \pm 0.76$ & $6.91 \pm 0.66$ & $6.77 \pm 0.77$ & $6.25 \pm 0.40$ \\
\hline GK & 7 & $15.396 .26 \pm 4.6 * *$ & $15.53 \pm 4.12^{* *}$ & $19.37 \pm 5.90 * *$ & $23.23 \pm 8.24 * * *$ & $20.03 \pm 6.38 * *$ & $21.20 \pm 7.68^{* *}$ \\
\hline metformin & 7 & $15.01 \pm 3.43^{* *}$ & $10.50 \pm 4.97 * \Delta$ & $11.72 \pm 4.67 * \Delta$ & $11.90 \pm 2.6 \mathrm{I}^{* \Delta \Delta}$ & $9.93 \pm 5.25 * \Delta \Delta$ & $10.44 \pm 3.84^{* \Delta \Delta}$ \\
\hline YNJH & 7 & $|5,27 \pm 3.2|^{* *}$ & $16.6 \pm 6.79 * *$ & $16.93 \pm 4.95 * *$ & $17.52 \pm 8.77^{* *}$ & $16.37 \pm 7.04 * * \Delta$ & $14.44 \pm 6.48^{* * \Delta}$ \\
\hline YNJM & 7 & $15.07 \pm 3.27 * *$ & $17.24 \pm 9.00 * *$ & $13.40 \pm 4.13^{* * \Delta}$ & $|3.90 \pm 2.9| * * \Delta \Delta$ & $14.17 \pm 8.92^{* * \Delta}$ & $11.13 \pm 2.50 * \Delta \Delta$ \\
\hline YNJL & 7 & $15.09 \pm 3.24 * *$ & $23.33 \pm 10.04 * * * \Delta$ & $17.87 \pm 3.79 * *$ & $23.36 \pm 7.49 * * *$ & $21.50 \pm 8.29 * * *$ & $19.57 \pm 8.59 * * *$ \\
\hline
\end{tabular}

Notes: ${ }^{*} p<0.05$ or ${ }^{* *} p<0.01$ compared to control. ${ }^{\Delta} p<0.05$ or ${ }^{\Delta \Delta} p<0.01$ compared to GK group at the same time point. 


\section{CON}

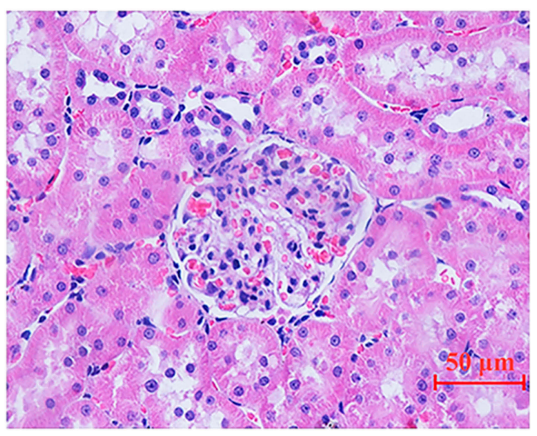

YNJH

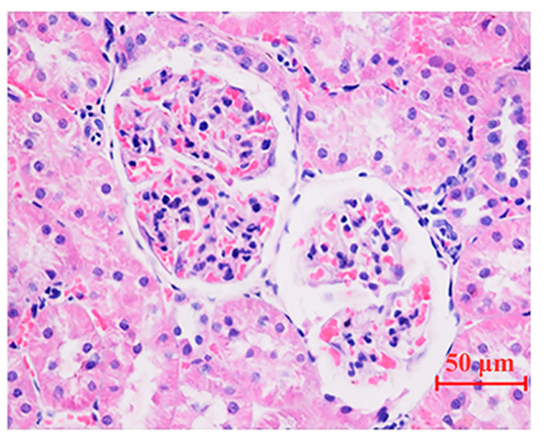

GK

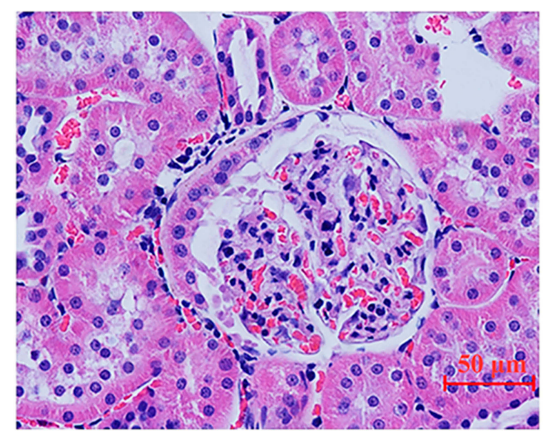

YNJM

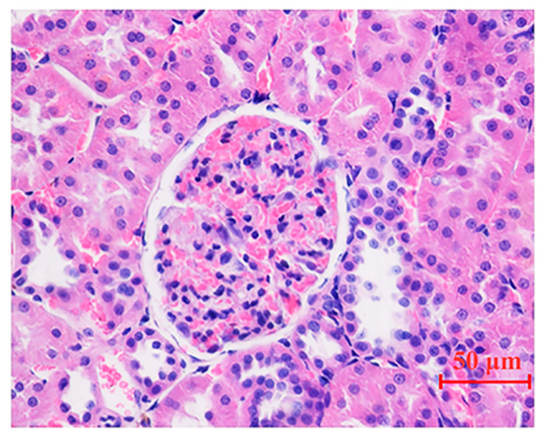

metformin

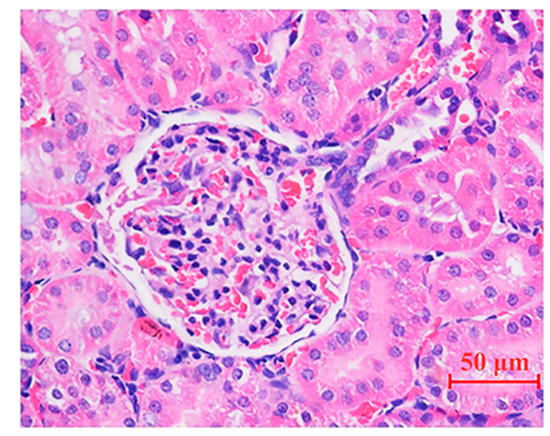

YNJL

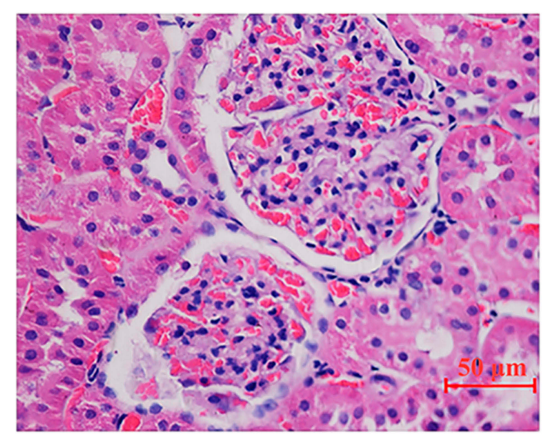

Figure I YNJ ameliorated renal cortex injury of diabetes rats detected by HE staining. YNJH (high dose): $12.5 \mathrm{~g} \mathrm{~kg}^{-1} \mathrm{~d}^{-1} \mathrm{YNJM}$ (medium dose): $6.25 \mathrm{~g} k \mathrm{~g}^{-1} \mathrm{~d}^{-1} \mathrm{YNJL}$ (low dose): $3.125 \mathrm{~g} \mathrm{~kg}^{-1} \mathrm{~d}^{-1}$.

CON

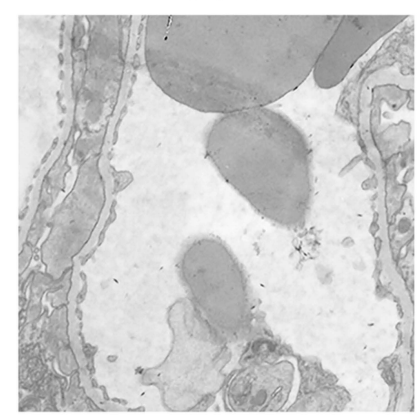

GK C

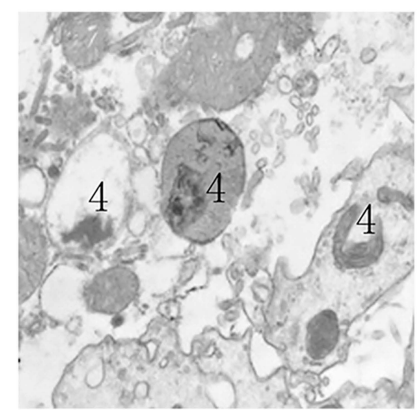

GK A

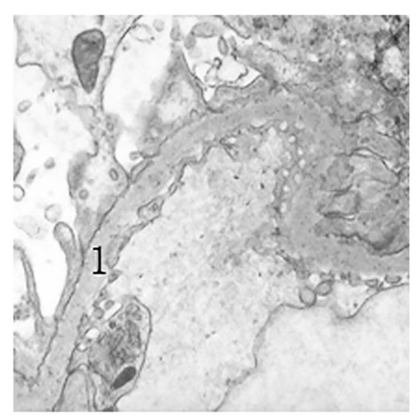

YNJM-D

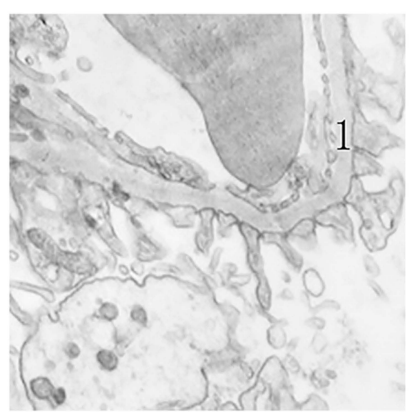

GK B

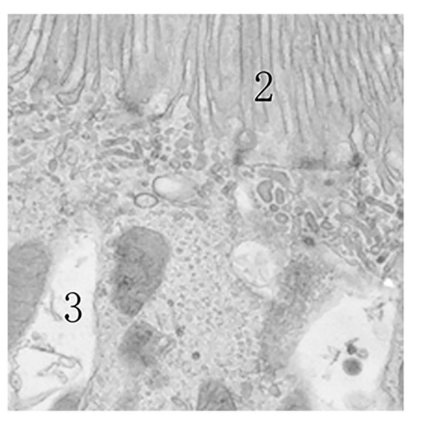

YNJM-E

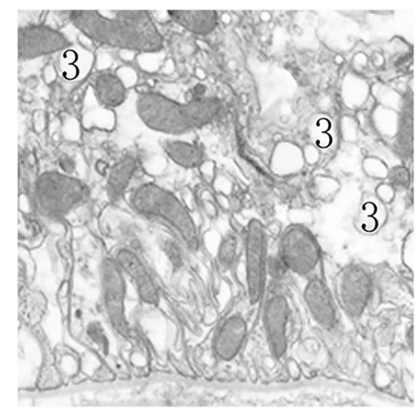

Figure 2 Electron microscope presented renal ultrastructure in different groups.I: basement-membrane. 2: microvillus. 3: Vacuolar structure. 4: autophagosome. CON:×3150; GK A, B×5000; GK C×4000; YNJM-D: ×6300; YNJM-E:×4000). 
Table 2 The Comparison of the Basal Membrane Thickness (Mean \pm SD)

\begin{tabular}{|l|l|l|}
\hline Group & Number & Thickness $(\boldsymbol{\mu m})$ \\
\hline CON & 7 & $0.145 \pm 0.028$ \\
Model & 7 & $0.312 \pm 0.048^{\Delta}$ \\
YNJM & 7 & $0.231 \pm 0.024^{\Delta}$ \\
\hline
\end{tabular}

Notes: ${ }^{\Delta}$ compared to $p<0.05$, *compared to model, $p<0.05$.

renal tubules, but the microvilli were more orderly arranged (Figure 2). In the YNJM group, basal membrane thickness was significantly reduced than GK group (Table 2). However, there were still many vacuoles in the cytoplasm of the renal tubular epithelial cells, suggesting that YNJM could improve the ultrastructure of the GK rats' kidneys (Figure 2).

\section{Yu Nu Compound Significantly Decreased Cell Autophagy and Apoptosis}

The results of WB analysis indicated that the expression of proteins mediating autophagy LC3 II, Atg5, Atg12 and
Beclin1 in GK group was significantly reduced comparing to Model (Figure 3A-B). Furthermore, the ratio of LC3-II/LC3-I was significantly decreased in GK group. Therefore, autophagy activities of renal cortex could be markedly reduced by YNJM. The proteins (Bax, cleaved-Caspase3) promoting apoptosis in GK group were markedly increased comparing to Model. Anti-apoptosis protein Bcl-2 showed increasing expression (Figure 3B). Moreover, TUNNEL staining showed that cell apoptosis was significantly reduced by YNJM administration in GK rats comparing to model group (Figure 3C). mTOR mRNA and mTOR levels were significantly increased by YNJ treatment (Figure 3A-B).

\section{Yu Nu Compound Significantly Promoted Proliferation and Reduced Apoptosis in Podocytes Exposed to HG}

CCK8 assay showed that the YNJ of medium dose had better effects on promoting proliferation (Figure 4A). Furthermore, the apoptosis levels were most significantly decreased by YNJM (Figure 4B-C). The result indicated that the YNJ of medium dose had better protective effects than other doses.

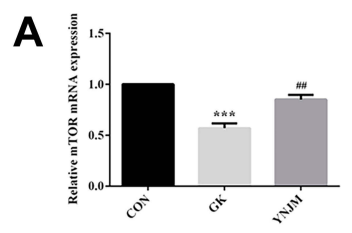

C
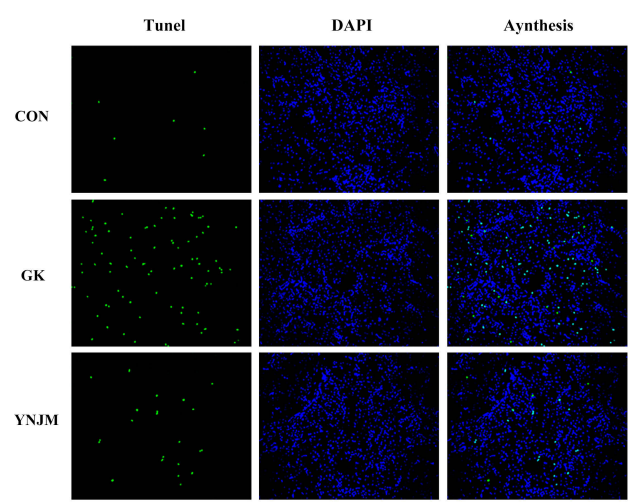

B
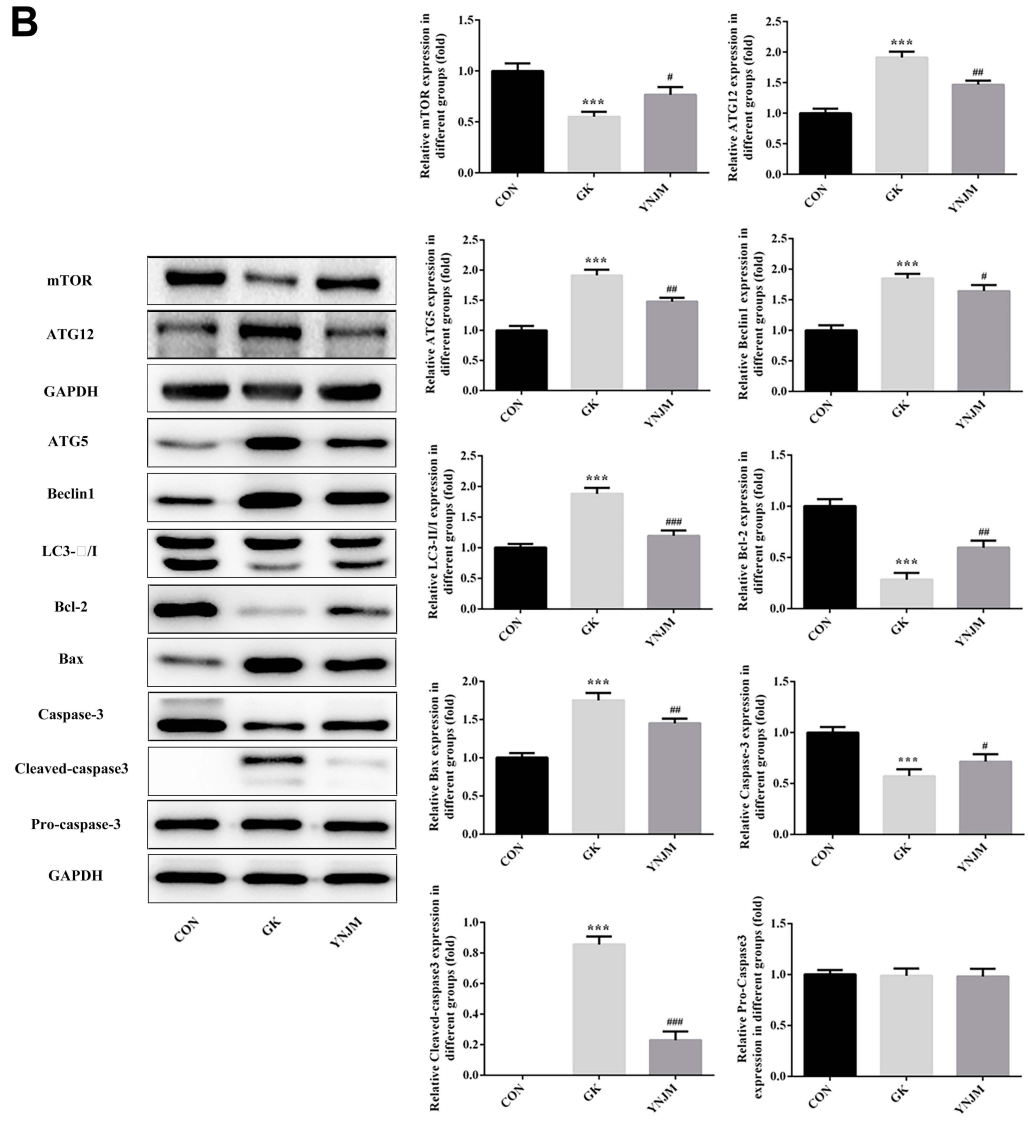

Figure 3 (A-B) The autophagy and apoptosis-related proteins were detected by WB or RT-qPCR. Data were shown as mean \pm SD. (C) YNJ reduced renal cells apoptosis detected by TUNNEL staining. ${ }^{* * *} p<0.001$ comparing to CON group. ${ }^{\#} p<0.05,{ }^{\#} p<0.01$ or ${ }^{\# \#} p<0.001$ comparing to GK group. 
A

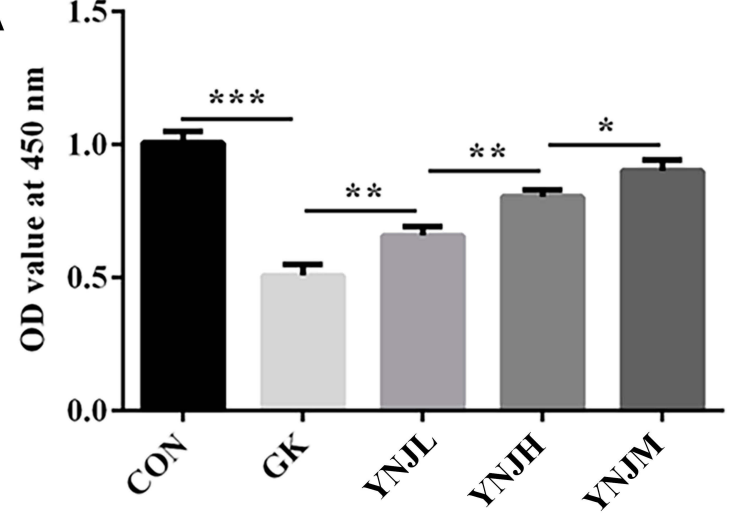

C

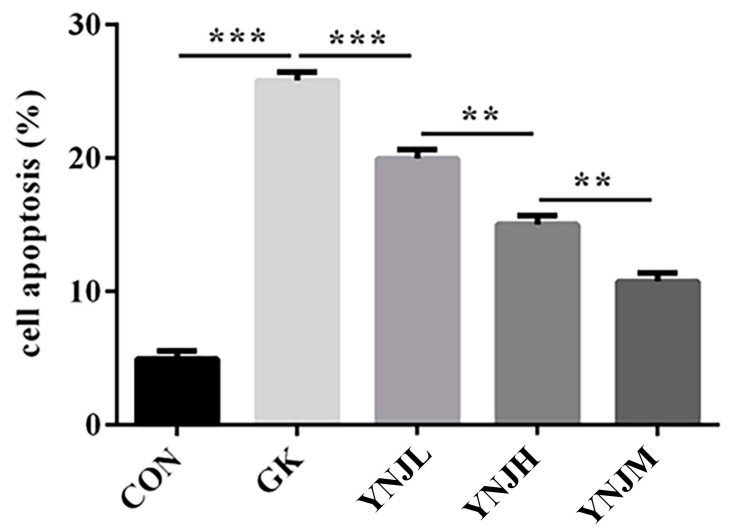

B

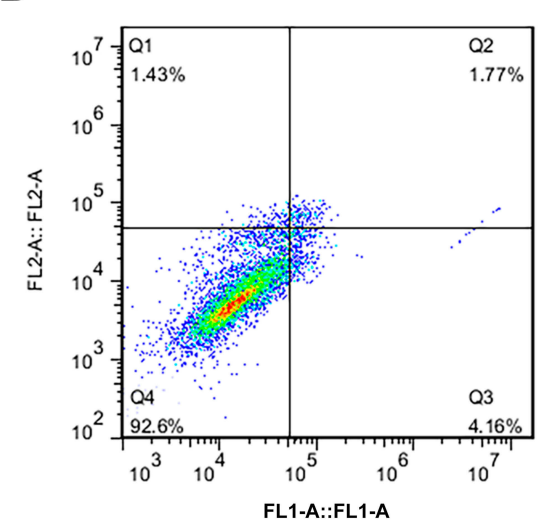

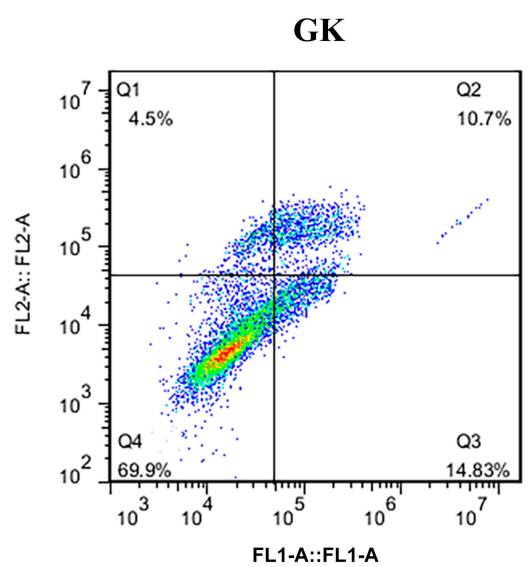

YNJL

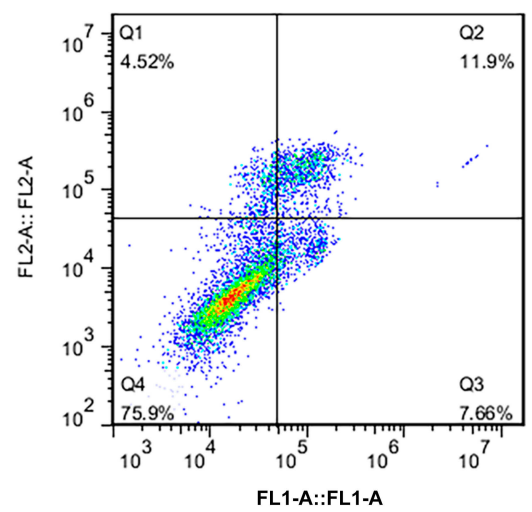

YNJH
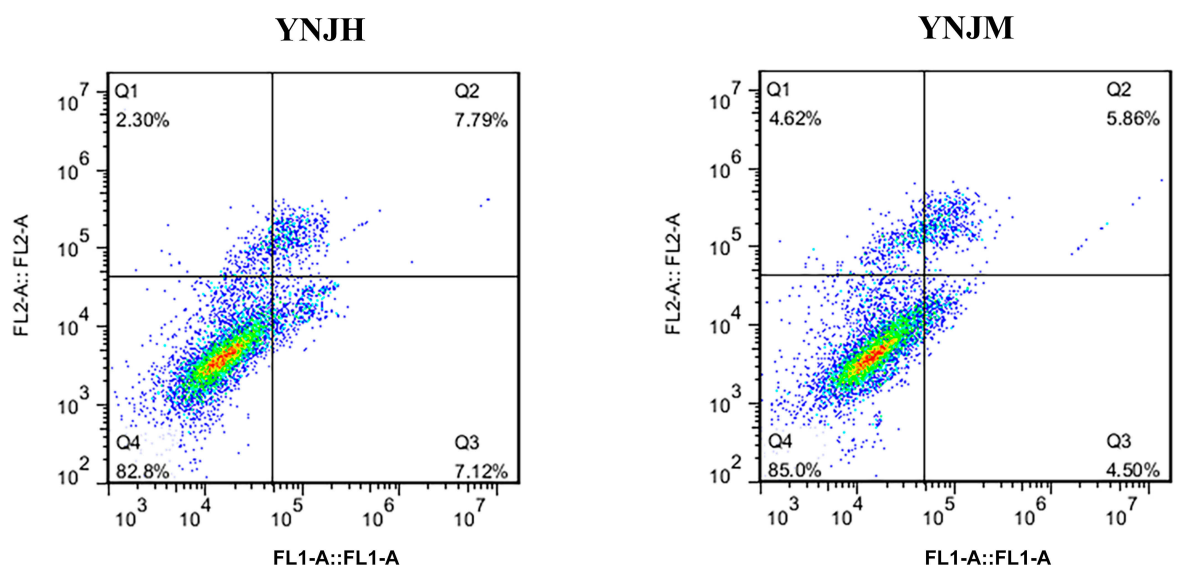

Figure 4 (A-C) Yu Nu compound significantly promoted proliferation and suppressed apoptosis which, respectively, were analyzed by CCK8 assay and flow cytometry. Data were shown as mean \pm SD. $* p<0.05$, $* * p<0.01$ or $* * * p<0.00$ I.

\section{Yu Nu Compound Markedly Regulated Cell Autophagy and Apoptosis Pathway}

The expression of autophagy-related proteins and apoptosis-related proteins was detected by WB (Figure $5 \mathrm{~A}-\mathrm{C}$ ). The results displayed that YNJ significantly decreased the ratio ofLC3- II/LC3- I, accompanied by the decrease of Atg5, Atg12 and Beclin1 compared with Model group.
The medium dose exhibited better inhibitory effects than other doses (Figure 5A-C). The results of apoptosisrelated proteins analysis implied that YNJ inhibited podocytes apoptosis possibly through suppressing mitochondrial-mediated apoptosis pathway. After YNJ treatment, mTOR was significantly increased comparing to Model (Figure 5C). 
A
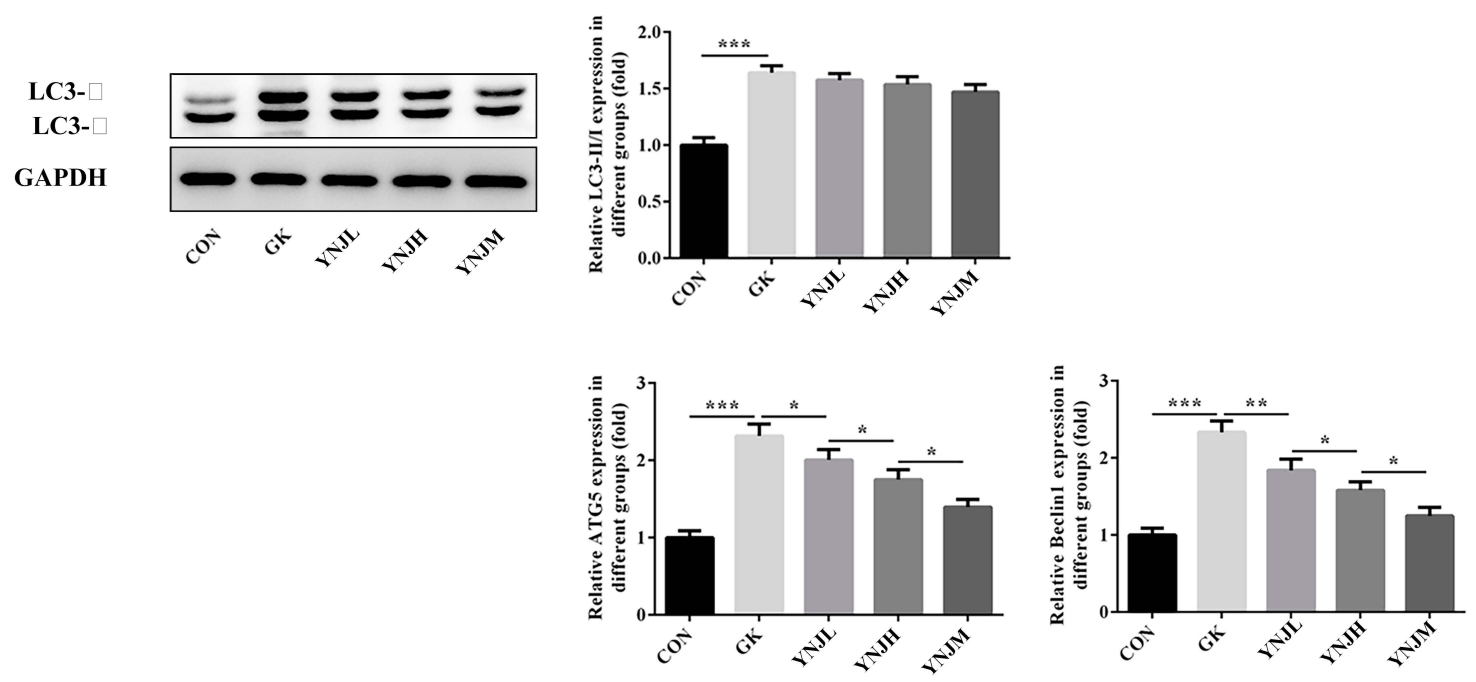

B
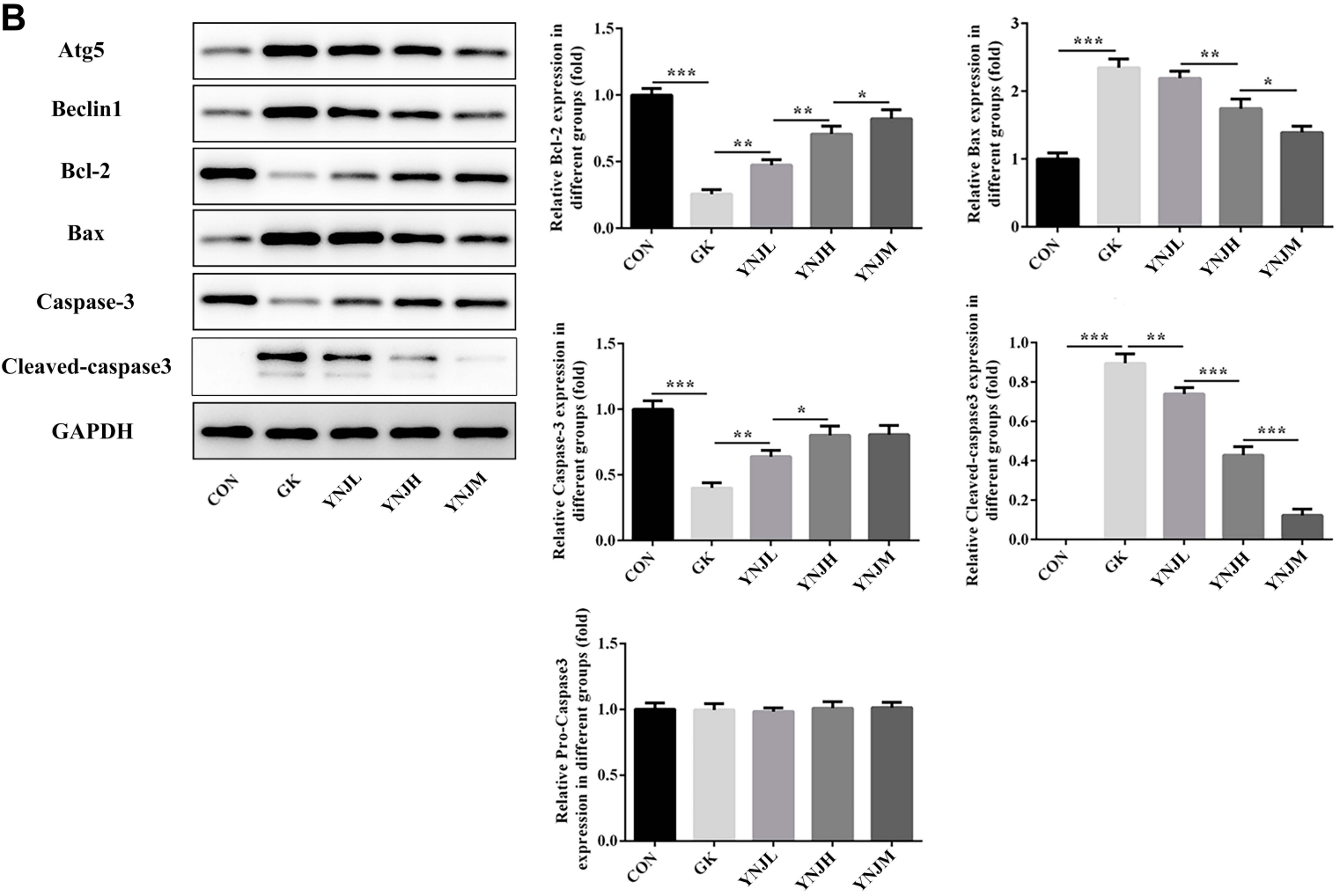

C
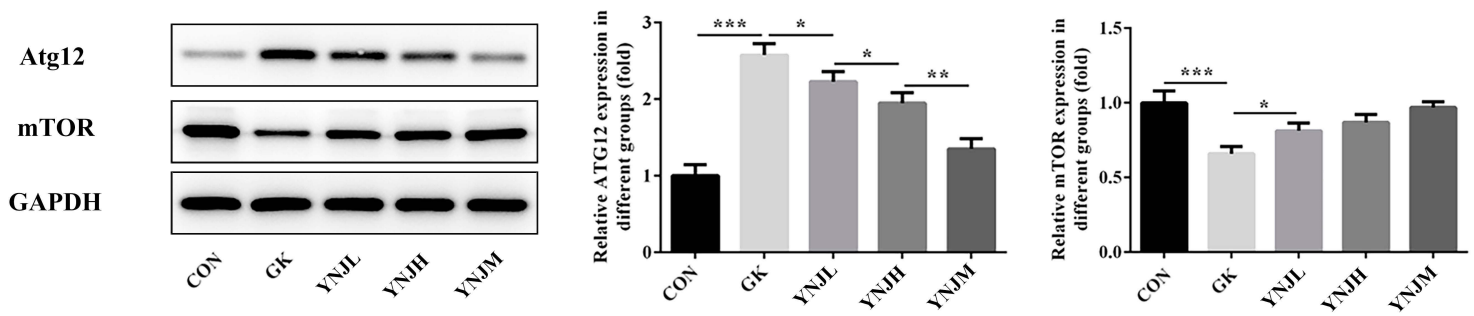

Figure 5 (A-C) Yu Nu compound affected the expression of autophagy-related and apoptosis-related proteins analyzed by Western blots. Data were shown as mean \pm SD. $*_{p}<0.05, * * p<0.01$ or $* * * p<0.001$. 


\section{Yu Nu Compound Affected Podocyte Apoptosis and Autophagy via Regulating mTOR}

The aforementioned results suggested that YNJ with medium dose had relatively better protection effect than YNJ with low or high dose. Therefore, the YNJ with medium dose was utilized for subsequent transfection experiment. qPCR evaluated the transfection efficacy of plasmids interfering mTOR expression. The result showed that shRNA -mTOR-1 had obvious effects on suppressing the expression of mTOR than shRNA-mTOR-2 (Figure 6A). The decrease of endogenous mTOR expression markedly reversed the effects of YNJ on podocytes proliferation, apoptosis and the ratio of LC3II/ I, which implied that the effects of YNJ on podocytes

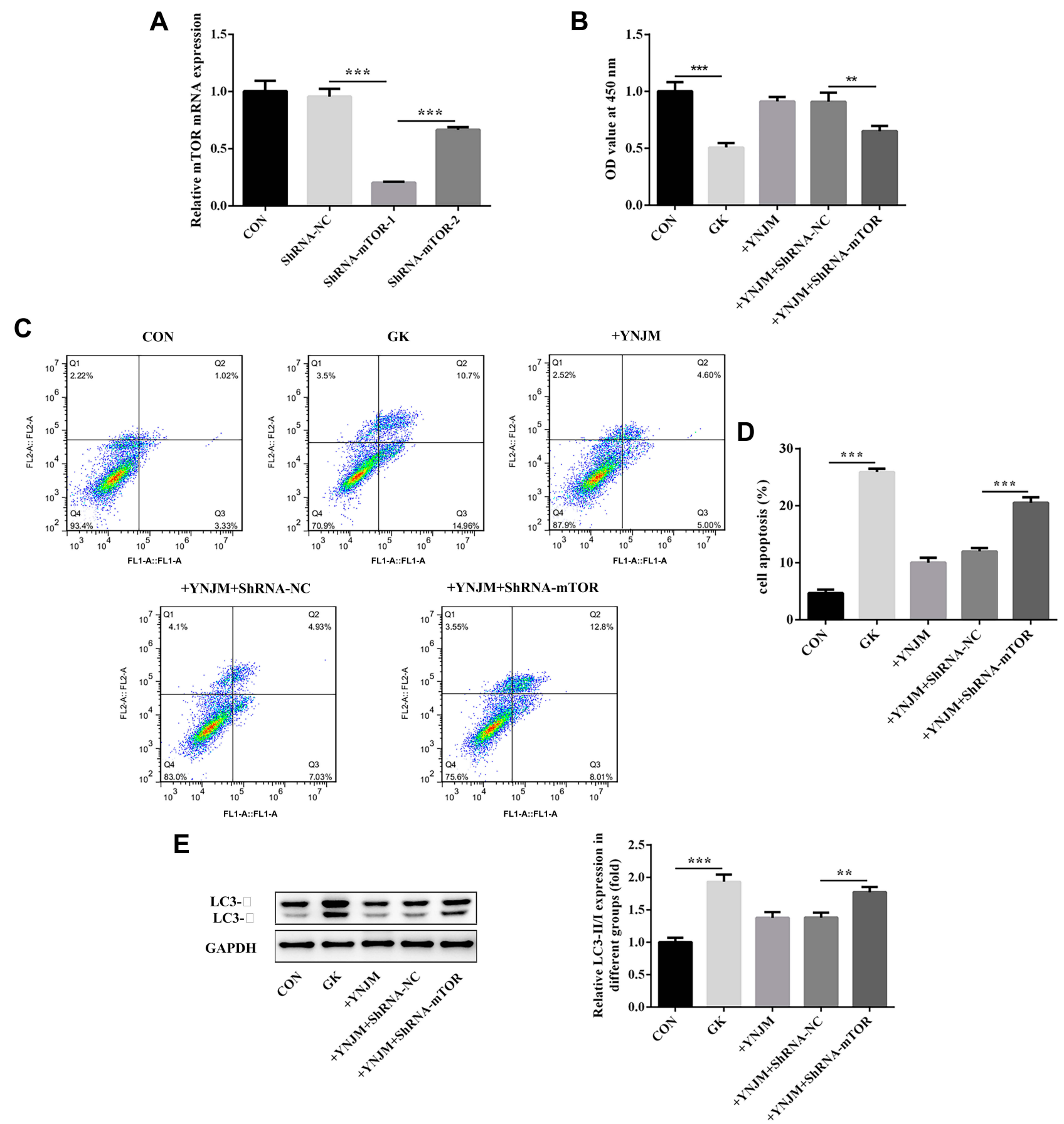

Figure 6 (A) qPCR was used to analyze transfection efficacy of shRNA-mTOR-I or shRNA-mTOR-2 plasmids. (B-D) mTOR knockdown reversed the effects of YNJ on podocytes proliferation and apoptosis separately analyzed by CCK8 assay or flow cytometry. (E) mTOR knockdown reversed the effects of YNJ on autophagy through analyzing the ratio of LC3-II/LC3-I which evaluated by Western blots. Data were shown as mean \pm SD. $* * p<0.0 \mathrm{I}$ or $* * * p<0.00 \mathrm{I}$. 
injury caused by HG could be attributed to the complexity of YNJ compound (Figure 6B-E).

\section{Discussion}

Our study shows that the medium dose of YNJ markedly ameliorates renal injury and decreases HG levels in vivo study, which demonstrates the protective role of YNJ in renal injury in diabetic rat. In addition, YNJ significantly regulates cell autophagy and apoptosis in vivo. Furthermore, autophagosome in podocytes presents high levels in GK rats. Then, we found that high autophagy levels also appear in podocyte exposed to high glucose than control group. A study has shown that autophagy could be enhanced in diabetic models and high glucose enhances autophagy flux in podocytes. ${ }^{18}$

Podocytes possess high autophagy levels in normal physiological conditions. ${ }^{19}$ However, whether the autophagy activation could induce cell injury depending on the intervention. Our study showed that YNJ significantly decreased autophagy, which indicated that YNJ could maintain a moderate autophagy level in DN through mTOR pathway in vivo and vitro. Although HG-induced podocytes autophagy exerts protective effects, ${ }^{20}$ podocyte damage can still not be reversed. Persistent presence of pathological factors contributes to the continuous increase of autophagy level, which may break the balance of autophagy in podocytes and lead to excessive autophagy, eventually causing programmed death of podocytes autophagy, ${ }^{21} \mathrm{~A}$ review shows that high basal autophagy is necessary for maintenance of podocytes function. ${ }^{5}$ Due to the complexities of compound in traditional Chinese medicine, the pathologic process of diabetes can be interfered through the interaction of various mechanisms, which could avoid further damage caused by overuse or underuse of a single target. ${ }^{22,23}$ Therefore, YNJ could reduce podocytes injury through multiple signally pathway to avoid high autophagy beyond basal autophagy levels in podocytes exposed to high glucose.

The microtubule-associated protein 1 light chain 3 (LC3), belin-1 and autophagy-related proteins (Atg) are involved in forming autophagosomes. ${ }^{24}$ The ratio of LC3II and LC3-I reflects levels of the autophagy flux or the autolysosomal degradation that is blocked. ${ }^{25}$ Our study discovered that the ratio of LC3-II/LC3-I was significantly decreased in podocytes exposed to HG followed by YNJ treatment. Therefore, the autophagy activities of podocytes induced by HG might be notably increased by YNJ. The study has demonstrated that streptozotocin promotes LC3 expression not due to a blockade in autophagy influx in diabetes, which indicates increased autophagy activities in early diabetes. ${ }^{18}$ Podocytes autophagy has been reported to exert protective effects under HG stimuli. ${ }^{26}$ However, the time of HG-induced autophagy shows differences in different podocytes lines. ${ }^{18,27}$ However, autophagy beyond a certain degree possibly accelerated cell apoptosis. In our study, YNJ markedly increases anti-apoptosis protein Bcl2 and reduces pro-apoptosis protein Bax and cleaved-caspase3 levels. Once the cell is induced by apoptotic factors, Bcl-2 family proteins could be transferred to the mitochondria, leading to the release of the dying factors containing Caspase3. ${ }^{11,28,29}$ Thus, YNJ notably decreases cell apoptosis partly through regulating mitochondria-dependent apoptotic pathway.

Glycation end metabolites increase protein kinase activity and activate polyol pathway, which have been identified as the pathogenesis of classic diabetic nephropathy. Increased advanced glycation end products, protein kinase activities and activation of the polyol pathway are considered as classical pathogenesis of DN. ${ }^{30}$ In addition, cell stress response including oxidative stress and endoplasmic reticulum stress, and mammalian target of rapamycin (mTOR), AMP-activated protein kinase (AMPK) pathway are involved in the development of DN as well, which is thought as important causes of autophagy formation. ${ }^{27,31,32}$ mTOR pathway is involved in regulating podocytes apoptosis and autophagy in DN cell models. ${ }^{15,33,34}$ Furthermore, mTOR dysregulation can promote glomerular diseases. ${ }^{33}$ Our study shows that YNJ can maintain mTOR expression at a normal level in vitro and vivo. Thus, YNJ regulates podocytes apoptosis and autophagy possibly through maintaining normal mTOR expression.

DN could be ascribed to abnormal hemodynamics and metabolism caused by HG stimulation. All in all, our study indicated that the complexity of YNJ compound could reduce podocytes injury through acting on diverse targets and maintain moderate autophagy activities to avoid excess cell apoptosis through mTOR pathway.

\section{Acknowledgments}

This work was supported by the National Natural Science Foundation of China (No.81774144, No.81703909) and the Nature Science Foundation of Fujian Province (No.2018J0188) 


\section{Disclosure}

The authors declared no conflict of interest.

\section{References}

1. Yu L, Haoyu W, Jiahua H, Hao Z, Suiqiang F. Research progress on regulation of pancreatic $\beta$ cell autophagy by chinese medicine. Chinese MED MODERN DISTANCE EDUC CHINA. 2018;16 (10):156-158.

2. Caigu H, Changhui Q, Yvmei H, Xuehua Z. Autophagy and the pancreatic beta-cell in human type 2 diabetes. J Fujian Univ TCM February. 2014;24(01):11-14. doi:10.4161/auto.5.7.9511.

3. Marchetti P, Masini M. Autophagy and the pancreatic beta-cell in human type 2 diabetes. Autophagy. 2009;5(7):1055-1056. doi: doi:10.4161/auto.5.7.9511

4. Fang L, Zhou Y, Cao H, et al. Autophagy attenuates diabetic glomerular damage through protection of hyperglycemia-induced podocyte injury. PLoS One. 2013;8(4):e60546. doi:doi:10.1371/journal. pone.0060546

5. Hartleben B, Godel M, Meyer-Schwesinger C, et al. Autophagy influences glomerular disease susceptibility and maintains podocyte homeostasis in aging mice. J Clin Invest. 2010;120(4):1084-1096. doi:doi:10.1172/jci39492

6. Kitada M, Kume S, Takeda-Watanabe A, Kanasaki K, Koya D. Sirtuins and renal diseases: relationship with aging and diabetic nephropathy. Clin Sci. 2012;124(3):153-164. doi:doi:10.1042/cs20120190

7. He Y, Zhang M, Wu Y, et al. Aberrant activation of Notch-1 signaling inhibits podocyte restoration after islet transplantation in a rat model of diabetic nephropathy. Cell Death Dis. 2018;9(10):950. doi:doi:10.1038/s41419-018-0985-z

8. Jones SA, Mills KH, Harris J. Autophagy and inflammatory diseases. Immunol Cell Biol. 2013;91(3):250-258. doi:doi:10.1038/icb.2012.82

9. Chaowen S High glucose induces autophagy in podocytes. Exp cell res. 2013;319(6):779-89.

10. Mizushima N, Levine B. Autophagy in mammalian development and differentiation. Nat Cell Biol. 2010;12(9:823-830. doi:doi:10.1038/ ncb0910-823.

11. Zhang XQ, Dong JJ, Cai T, Shen X, Zhou XJ, Liao L. High glucose induces apoptosis via upregulation of Bim expression in proximal tubule epithelial cells. Oncotarget. 8(15):24119-24129. doi: doi:10.18632/oncotarget.15491

12. Mizushima N, Yoshimori T, Levine B. Methods in mammalian autophagy research. Cell. 2010;140(3):0-326.

13. Candeias E, Sebastiao I, Cardoso S, et al. Brain GLP-1/IGF-1 signaling and autophagy mediate exendin-4 protection against apoptosis in type 2 diabetic rats. Mol Neurobiol. 2018;55(5):4030-4050. doi: doi:10.1007/s12035-017-0622-3

14. Xu J, Deng Y, Wang Y, Sun X, Chen S, Fu G. SPAG5-AS1 inhibited autophagy and aggravated apoptosis of podocytes via SPAG5/AKT/ mTOR pathway. Cell Prolif. 2020;53(2):e12738. doi:doi:10.1111/ cpr.12738

15. Li C, Guan XM, Wang RY, et al. Berberine mitigates high glucoseinduced podocyte apoptosis by modulating autophagy via the mTOR/ P70S6K/4EBP1 pathway. Life Sci. 243. 117277. doi:10.1016/j. Ifs. 2020.117277

16. Zheng D, Tao M, Liang X, Li Y, Jin J, He Q. p66Shc regulates podocyte autophagy in high glucose environment through the Notch-PTEN-PI3K/Akt/mTOR pathway. Histol Histopathol. 2019;25:18178. doi:doi:10.14670/hh-18-178

17. Zhou J, Tan SH, Codogno P, Shen HM. Dual suppressive effect of MTORC1 on autophagy: tame the dragon by shackling both the head and the tail. Autophagy. 2013;9(5):803-805. doi:doi:10.4161/auto.23965
18. Lenoir O, Jasiek M, Henique C, et al. Endothelial cell and podocyte autophagy synergistically protect from diabetes-induced glomerulosclerosis. Autophagy. 2015;11(7):1130-1145. doi:doi:10.1080/ 15548627.2015.1049799

19. Kim WY, Nam SA, Song HC, et al. The role of autophagy in unilateral ureteral obstruction rat model. Nephrology. 2012;17 (2):148-159. doi:doi:10.1111/j.1440-1797.2011.01541.x

20. Liu Y, Zhang J, Wang Y, Zeng X. Apelin involved in progression of diabetic nephropathy by inhibiting autophagy in podocytes. Cell Death Dis. 8(8):e3006. doi:doi:10.1038/cddis.2017.414

21. Tharaux PL, Huber TB. How many ways can a podocyte die? Semin Nephrol. 2012;32(4):394 404. doi:doi:10.1016/j.semnephrol.2012. 06.011

22. Wang Y, Fan X, Qu H, Gao X, Cheng Y. Strategies and techniques for multi-component drug design from medicinal herbs and traditional Chinese medicine. Curr Top Med Chem. 2012;12(12):1356-1362. doi:doi:10.2174/156802612801319034

23. Ji T, Su SL, Zhu Y, et al. The mechanism of mulberry leaves against renal tubular interstitial fibrosis through ERK1/2 signaling pathway was predicted by network pharmacology and validated in human tubular epithelial cells. Phytother Res. 2019;33(8):2044-2055. doi: doi:10.1002/ptr.6390

24. Boya P, Reggiori F, Codogno P. Emerging regulation and functions of autophagy. Nat Cell Biol. 2013;15(7:713-720. doi:doi:10.1038/ncb2788.

25. Tanida I, Ueno T, Kominami E. LC3 and autophagy. Methods Mol Biol. 2008;445:77-88. doi:doi:10.1007/978-1-59745-157-4_4

26. Wu F, Li S, Zhang N, et al. Hispidulin alleviates high-glucoseinduced podocyte injury by regulating protective autophagy. Biomed Pharmacother. 2018;104:307-314. doi:doi:10.1016/j.biopha.2018. 05.017

27. Lim JH, Kim HW, Kim MY, et al.Cinacalcet-mediated activation of the CaMKKbeta-LKB1-AMPK pathway attenuates diabetic nephropathy in $\mathrm{db} / \mathrm{db}$ mice by modulation of apoptosis and autophagy. Cell Death Dis.;9(3):270. doi:10.1038/s41419-018-0324-4

28. Said RS, Mohamed HA, Kamal MM Coenzyme Q10 mitigates ionizing radiation-induced testicular damage in rats through inhibition of oxidative stress and mitochondria-mediated apoptotic cell death. Toxicol Appl Pharmacol. 383:114780. doi:10.1016/j.taap.2019. 114780

29. Nechushtan A, Smith CL, Lamensdorf I, Yoon SH, Youle RJ. Bax and Bak coalesce into novel mitochondria-associated clusters during apoptosis. J Cell Biol. 153(6):1265-1276. doi:doi:10.1083/ jcb.153.6.1265

30. Zhao X, Chen Y, Tan X, et al. Advanced glycation end-products suppress autophagic flux in podocytes by activating mammalian target of rapamycin and inhibiting nuclear translocation of transcription factor EB. J Pathol. 2018;245(2):235-248. doi:doi:10.1002/ path. 5077

31. Ravindran S, Kuruvilla V, Wilbur K, Munusamy S. Nephroprotective effects of metformin in diabetic nephropathy. $J$ Cell Physiol. 2017;232(4):731-742. doi:doi:10.1002/jcp.25598

32. Wu J, Zhang R, Torreggiani M, et al. Induction of diabetes in aged C57B6 mice results in severe nephropathy: an association with oxidative stress, endoplasmic reticulum stress, and inflammation. Am J Pathol. 2010;176(5):2163-2176. doi:doi:10.2353/ajpath.2010.090386

33. Godel M, Hartleben B, Herbach N, et al. Role of mTOR in podocyte function and diabetic nephropathy in humans and mice. J Clin Invest. 2011;121(6):2197-2209. doi:doi:10.1172/jci44774

34. Guzman J, Jauregui AN, Merscher-Gomez S, et al. Podocyte-specific GLUT4-deficient mice have fewer and larger podocytes and are protected from diabetic nephropathy. Diabetes. 2014;63(2):701-714. doi:doi:10.2337/db13-0752 


\section{Publish your work in this journal}

Diabetes, Metabolic Syndrome and Obesity: Targets and Therapy is an international, peer-reviewed open-access journal committed to the rapid publication of the latest laboratory and clinical findings in the fields of diabetes, metabolic syndrome and obesity research. Original research, review, case reports, hypothesis formation, expert opinion and commentaries are all considered for publication. The manuscript management system is completely online and includes a very quick and fair peer-review system, which is all easy to use. Visit http://www.dovepress.com/testimonials.php to read real quotes from published authors.

Submit your manuscript here: https://www.dovepress.com/diabetes-metabolic-syndrome-and-obesity-targets-and-therapy-journal 